\title{
Performance Comparison among (Star, Tree and Mesh) Topologies for Large Scale WSN based IEEE 802.15.4 Standard
}

\author{
Ziyad Khalaf Farej, PhD \\ Dep. of Medical Instrumentation Eng. \\ Northern Technical University \\ Technical Engineering College \\ Mosul, Iraq
}

\author{
Ali Maher Abdul-Hameed \\ Computer Engineering Department \\ Northern Technical University \\ Technical Engineering College \\ Mosul, Iraq
}

\begin{abstract}
This paper investigates the applicability of star, tree and mesh topology schemes for large scale Wireless Sensor Network (WSN) complying with IEEE 802.15.4 standard. The main focus of this work is to evaluate the performance of such network through simulation which is carried out via the discrete event OPNET simulator (version 14.5). The performance metrics of interest include throughput, end to end delay and packet drop rates. Performance comparison and analyses of different topologies have been made, and it is concluded that the selection of topology scheme depends on the application context.
\end{abstract}

\section{Keywords}

WSN, OPNET, IEEE 802.15.4, Performance Analysis.

\section{INTRODUCTION}

The IEEE 802.15.4 protocol has recently been adopted as a communication standard for low data rate, low power consumption and low cost Wireless Personal Area Networks (WPAN). This protocol is very flexible for a wide range of applications if appropriate tuning of its parameters is carried out [1]. WSN are often used for real-time applications, such as environment surveillance, medical care, and vehicle traffic control. In these contexts, in spite of the resource limitations that characterize the sensor nodes, WSNs have to provide a reliable coverage of the area of interest as well as to meet timing constraints [2]. In the IEEE 802.15.4 standard, all sensor nodes uses the random access protocol known as the CSMA/CA (Carrier Sense Multiple Access/ Collision Avoidance) medium access control protocol to transmit data to the master node. The advantage of a random access protocol is the simplicity of its implementation, lower system cost, and offers a low delay and reliable data transmission. Zigbee devices can transmit up to $250 \mathrm{kbps}$ at $2.4 \mathrm{GHz}$ which is sufficient data rate for typical Wireless Body Area Network (WBAN) applications. Each node will encapsulate its sensor data into an 802.1.5.4 MAC frame and transmit it to the master node [3].

In [4], the IEEE 802.15.4 performance is analyzed for Wireless Body Area Network (WBAN). The analysis focused on the long term power consumption of the sensors and presented a star network configuration for a body area network consisting of 10 body implanted sensors. In [5], the performance of IEEE 802.15.4 is analyzed based on OPNET simulator. The simulation result indicates the influence of ACK mechanism and different network load on the system performance, i.e. end-to-end delay, packet reception ratio and throughput of node, which provides an important theoretical basis for the construction of actual network. In [6], evaluation and comparison of IEEE 802.15.4 standard performance using Omnet++ simulator is performed with focusing on single sink scenario in terms of data delivery rate, goodput, throughput and error rate metrics.

This paper presents a comparison between the performances of star, tree and mesh topologies for the IEEE 802.15.4 standard based wireless sensor network when a large scale topology is needed in order to measure the impact of increasing number of nodes on throughput, end to end delay, packet drop rates. This work can be very helpful for those who want to deploy their sensor networks in the field of work and to predict the behavior of such a network.

The rest of the paper is organized as follows; In section II, a general overview of the IEEE 802.15.4 standard and its layers are given. In section III, the description of the network topologies is included. In section IV, a description of our OPNET model parameters is given. In section V, the modeled networks assumption is presented. In section VI, the evaluation of the performance results obtained from the OPNET simulations is included. In section VII, Concluding remarks are given.

\section{BRIEF OVERVIEW OF IEEE 802.15.4 \\ STANDARD}

The IEEE 802.15.4 standard (ZigBee) consists of four layers. The top two (Application and Network) layers specifications are provided by the ZigBee Alliance to provide manufacturing standards. The bottom two (Medium Access Control and Physical) layers specifications are provided by the IEEE 802.15.4-2006 standard to ensure coexistence without interference with other wireless protocols such as Wi-Fi [7].

\begin{abstract}
Application Layer This is the layer that makes the device useful to the user. For example, applications to monitor temperature, humidity, or any other desirable atmospheric parameters that can be placed on this layer for agricultural use. A single node can run more than one application. Applications are referenced with a number ranging from $1-240$. Meaning there is a maximum of 240 applications on a ZigBee device. Application number 0 is reserved for a unique application that exists on all ZigBee devices. The application number, 255, is also reserved to broadcast a message to all applications on a node.
\end{abstract}

Network Layer the self-healing mechanism, which is a feature of the standard, is acquired through this layer. As Figure 1 shows, this layer provides network and routing 
management, network message broker, and network security management.

Medium Access Control Sub-Layer is the layer extracted from the IEEE 802.15.4 standard and provides services to the network layer above. The MAC layer is responsible for data addressing to determine either where the frame is going, or coming from. The layer also provides multiple access control such as CSMA/CA allowing for reliable transfer of data. Beaconing is another feature implemented through this layer. Finally, the MAC sub-layer can be exploited by higher layers to achieve secure communication.

Physical Layer is provided by the IEEE 802.15.4 standard to manage the physical transmission of radio waves in different unlicensed frequency bands around the world and also to provide communication between devices within a WPAN: The bands are specified in the table 1, pairing it with the area that the band is used in. This layer allows for channel selection to avoid radio interference, as well as data exchange with the layer above (MAC sub-layer) providing it with service [8].

Table 1: IEEE 802.15.4 frequency bands

\begin{tabular}{|l|l|l|}
\hline $\begin{array}{l}\text { Frequency Range } \\
\text { (MHz) }\end{array}$ & $\begin{array}{l}\text { Numbers of } \\
\text { Channels Available }\end{array}$ & Region used \\
\hline $868-868.6$ & 1 & Europe \\
\hline $902-928$ & 10 & $\begin{array}{l}\text { North } \\
\text { America }\end{array}$ \\
\hline $2400-2483.5$ & 16 & Worldwide \\
\hline
\end{tabular}

\section{NETWORK TOPOLOGIES}

The ZigBee networks can contain a mixture of two potential components; FFD (Full Function Device) which can work as a coordinator or a router, and RFD (Reduced Function Device) which can work as an end device only, and these different types of nodes can have different roles within the network layer, but all various types can have the same applications [9].

\subsection{Star Topology}

In this topology, a coordinator is surrounded by a group of either end devices or routers. Even though routers are connected to the coordinator, their message relaying functions are not activated. This topology is attractive because of its simplicity, but at the same time have some disadvantages. In the event that the coordinator stops functioning, the entire network is functionless because all traffic must travel through the center of the star. For the same reason, the coordinator could easily be a bottleneck to traffic within the network, especially for large scale ZigBee network which may have more than hundred of nodes [10].

\subsection{Tree Topology}

In a tree network, a coordinator initializes the network, and is the top (root) of the tree.

The coordinator can now have either routers or end devices connected to it. For every connected router, more child nodes can connect to the router. Child nodes cannot connect to an end device because it does not have the ability to relay messages. This topology allows for different levels of nodes, with the coordinator being at the highest level. To pass messages to other nodes in the same network, the source node must pass the message to its parent, which is the node higher up by one level of the source node, and the message is continually relayed higher up in the tree until it reaches the destination node. Since the message can take only one potential paths, this type of topology is not the most reliable topology. If a router fails, then all its children are cut off from communicating with the rest of the network [10].

\subsection{Mesh Topology}

The mesh topology is the most flexible among topologies. Flexibility is present because a message can take multiple paths from source to destination. If a particular router fails, then ZigBee's self healing mechanism (make route discovery) will allow the network to search for an alternate path for the message to take [10].

\section{SIMULATION PARAMETERS CONFIGURATION OF THE OPNET NETWORK MODELS}

The network models that are used in the simulation for modeling the three topologies are shown in Figures $(2,3 \& 4)$ bellow:-

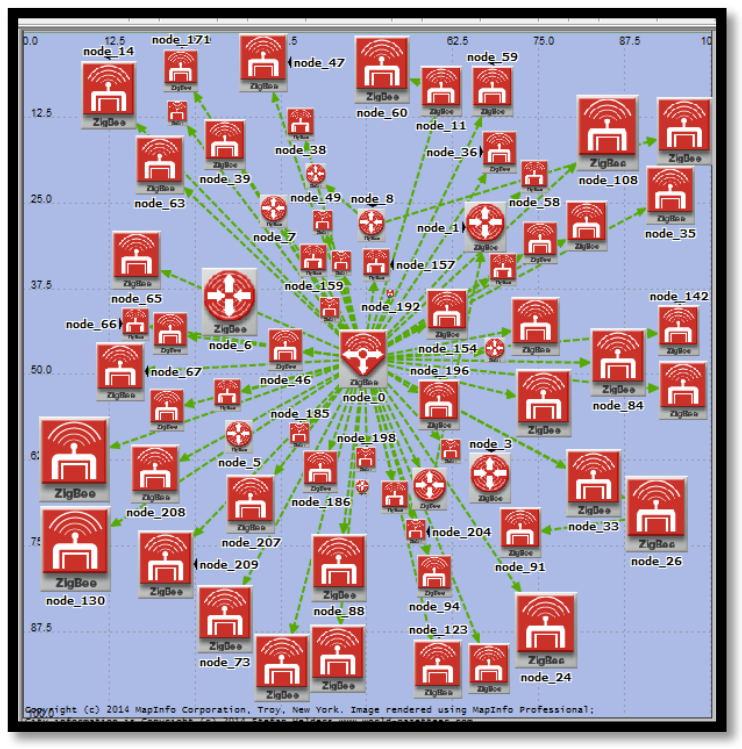

Figure 2: Mesh

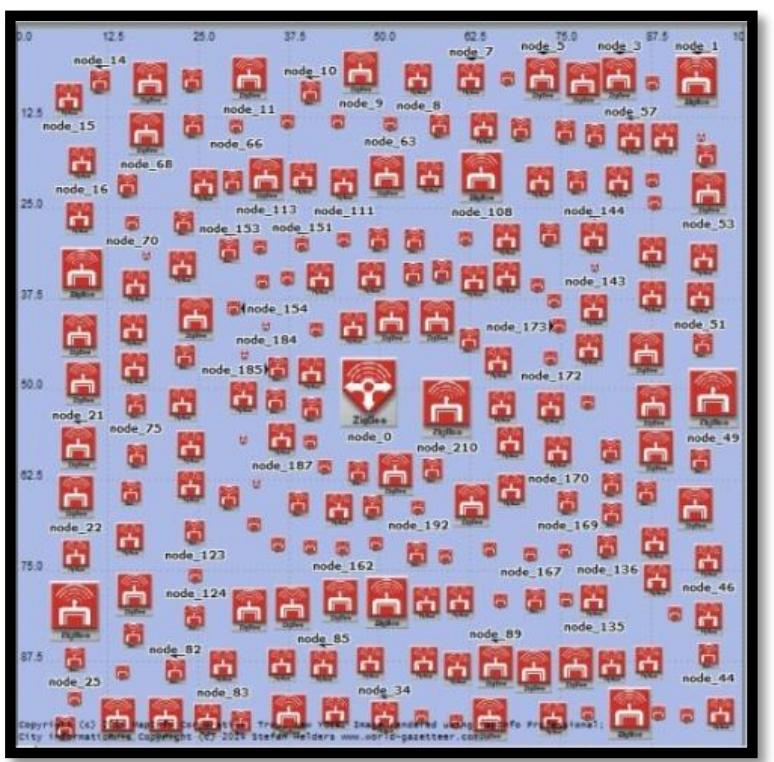

Figure 3: Star 


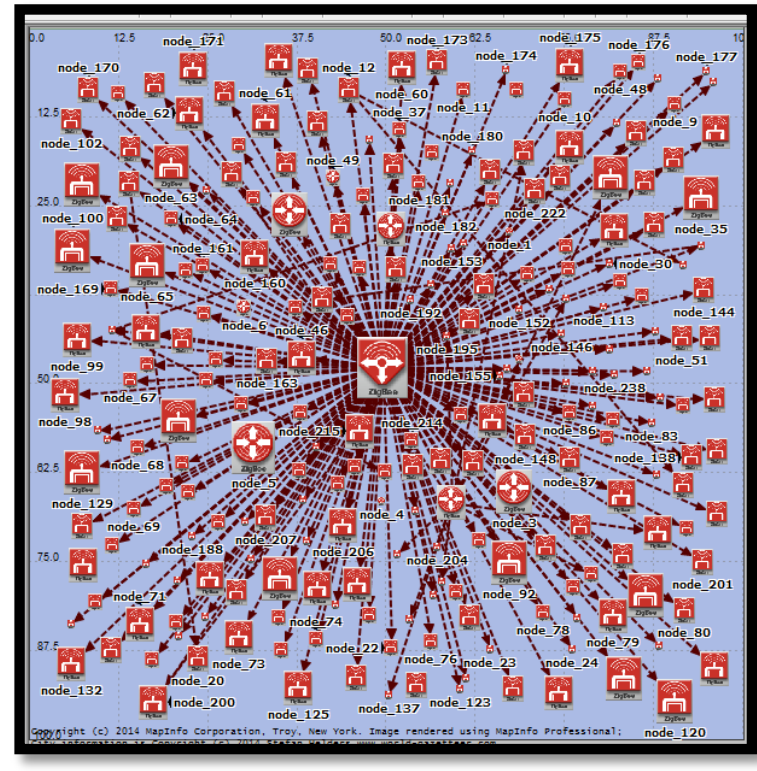

Figure 4: Tree

The parameters that are used in the setting of the OPNET models are shown in Tables 2 as follows:

Table 2: parameters setting

\begin{tabular}{|l|l|}
\hline Max. number of children & 255 \\
\hline Max. number of routers & 10 \\
\hline Max. depth & 10 \\
\hline Mesh routing & $\begin{array}{l}\text { Enable in mesh topology } \\
\text { only }\end{array}$ \\
\hline Destination & Random \\
\hline Packet interval time (sec) & Constant (1.0) \\
\hline Packet size (bits) & Constant (1408) \\
\hline Ack mechanism & Disable \\
\hline Minimum value of backoff & 3 \\
\hline Maximum number of backoff & 5 \\
\hline Channel sensing duration (sec) & 0.2 \\
\hline $\begin{array}{l}\text { Packets reception power } \\
\text { threshold (dbm) }\end{array}$ & -90 \\
\hline Transmit power (Watt) & 0.1 \\
\hline Transmission band & $2.4 \mathrm{GHz}$ \\
\hline
\end{tabular}

\section{MODELS ASSUMPTION}

The modeled networks are simulated under the following assumption:-

1- All system nodes distributed over $100 \mathrm{X} 100 \mathrm{~m}^{2}$ area.

2- ACK mechanism is not used.

3- Slotted CSMA/CA is not used.

4- $\quad$ All nodes are fixed.

5- Beacon-enabled mode is not used.

6- The addressing mechanism used in PANID is only 16 bit.

7- The destination is randomly chosen from their neighbors.

\section{RESULTS ANALYSIS AND \\ PERFORMANCE EVALUATION}

Performance comparison has been made among the (star, tree and mesh) topologies for different evaluation meters (packet drop, end to end delay and throughput) VS. number of nodes in the case of large scale as follows:

\subsection{Number of nodes vs. throughput}

The curves in Figure (5) below show throughput variation against number of nodes for the three topologies.

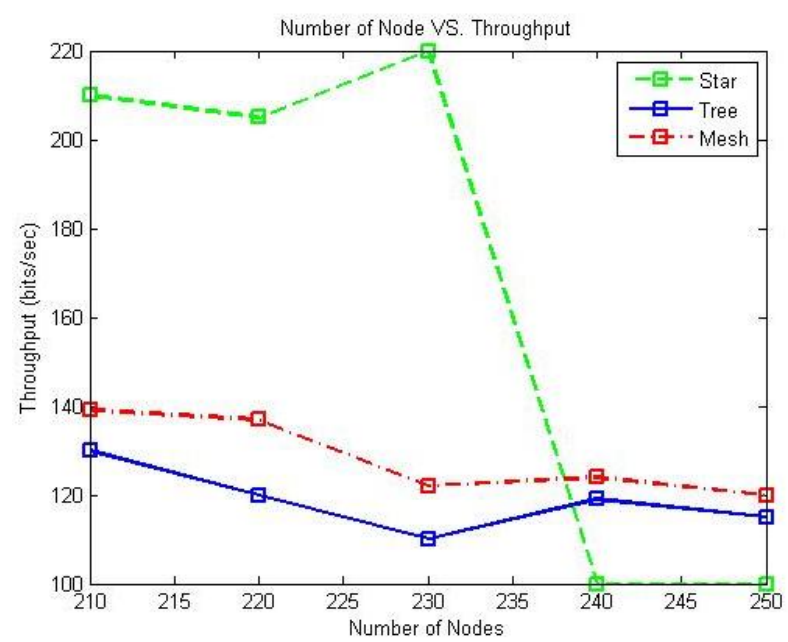

Figure 5: Global throughput variation

In general it is seen that increasing number of nodes causes the throughput to increase up to a certain value then it start to decrease. The reason behind that differs according to different topologies. In case of star topology the drop of throughput is quite obvious after 240 nodes and the reason for that is that increasing number of nodes causes an increase in packet collision as well as the fact that the star topology has a centralized connection therefore it may reach a bottle neck situation in a certain level and consequently leads to the drop of a certain number of packets. In the case of tree and mesh topologies, the throughput shows more stability. The reason behind that is that the connections in these topologies are decentralized because of the presence of routers between nodes and coordinator which leads to a state of load balance. The drop of throughput is due to collisions that happen at routers level but, in general, the mesh topology shows higher throughput than tree topology and its maximum gain value is $(9.821 \%)$ at 220 nodes.

\subsection{Number of nodes vs. packet drop}

Figure (6) shows the packet drop variation against number of nodes for the three topologies.

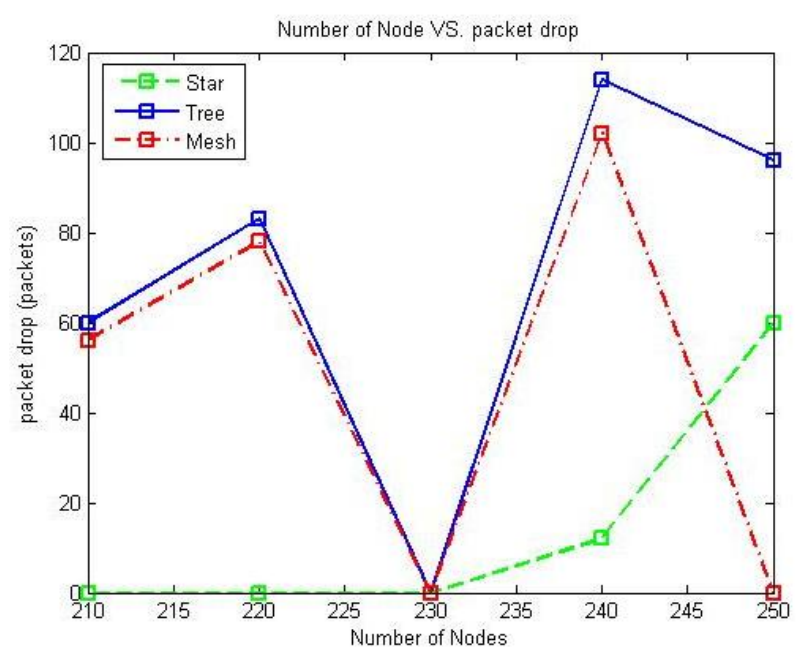

Figure 6: Packet drop variation 
It is seen that the least value of packet drop is in the case of star topology and it starts to increase by increasing number of nodes (more than 240 nodes) due to packet collision at the coordinator level which explains the sharp drop of throughput at 240 and 250 nodes. For tree and mesh topology it is seen that there is an obvious convergence in packet drop which happens mostly at router level when nodes try to send their information to their parent. It is worth to mention that the average packet drop in tree topology in higher by (34.072\%) than mesh topology which explains the higher throughput in case of mesh topology.

\subsection{Number of nodes VS. end to end delay}

Figure (7) shows the end to end delay variation against number of nodes for the three topologies.

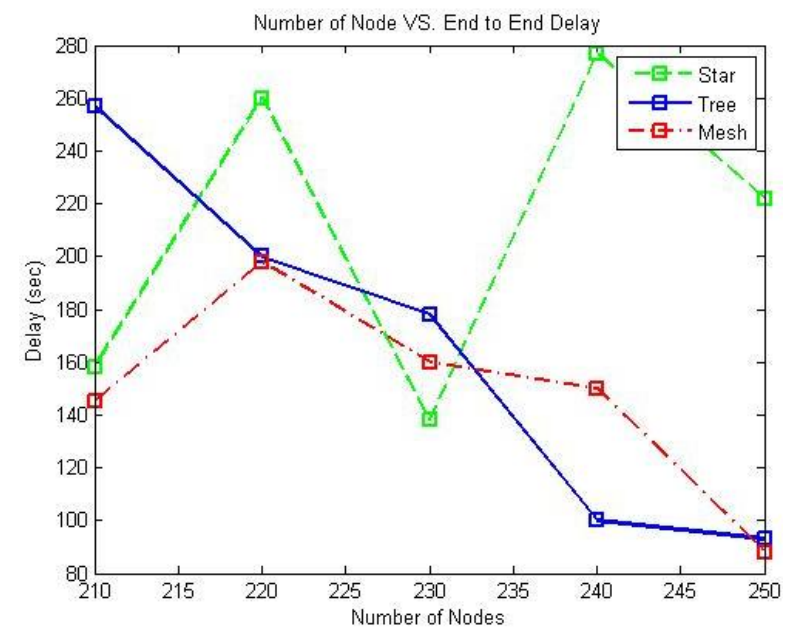

Figure 7: End to end delay variation

In general it is seen that the average end to end delay for star topology is tending to increase as the number of nodes increases beyond the optimum (at which the throughput is maximum) number of nodes. However the average delay for tree and mesh topologies is tending to decrease as the number of nodes increases beyond the optimum number of nodes at which the throughput is maximum. The reason behind that is the higher probability of packet collision caused by the random access mechanism (CSMA/CA) which occurs either at the coordinator only (for star topology) or at coordinator and routers when their child nodes try to access the channel and send their information. In comparison with star topology and up to (240) node number, this results in higher packet dropping probability for tree and mesh topologies and as a result throughput and end to end delay decreasing. Regarding the mesh and tree topologies, both of them show end to end delay increasing as the number of nodes increases to the optimum number of nodes (at which throughput is maximum), and end to end delay decreasing as the number of nodes increases beyond that optimum number; however in comparison with tree topology the mesh topology shows lower average end to end delay, and the reason for that is the mesh routing algorithms which facilitate the arrival of packets to coordinator i.e. it always tries to choose the less congested (shortest or fastest) route to reach the destination.

\section{CONCLUSION}

In this paper the designed models are simulated to trace the applicability of star, tree and mesh topology schemes for large scale WSNs. Performance comparison and analyses of different topologies is made in terms of throughput, end to end delay and packet drop rates. For a network with size of 250 nodes, it is found that up to around (230) nodes, the star topology is ideal for real time services, regarding the urgent healthcare signals, since it shows stable maximum throughput of $220 \mathrm{Kbit} / \mathrm{s}$ with zero number of packet dropping and minimum average end to end delay. For large number of nodes (230 to 250) networks, both tree and mesh topologies can be applied; however the mesh topology is more suitable than the tree topology and with less degree of real time application services. Such a study would be essential since throughput, end to end delay and nodes deployment in the field is very critical for WSNs.

\section{REFERENCES}

[1] Petr, J., Anis, K., Mário, A., Eduardo, T., Zdeněk, H., A Simulation Model for the IEEE 802.15.4 Protocol: Delay/Throughput Evaluation of the GTS Mechanism.

[2] Marco, G., Daniele, M., Alessandro, N., Carla-Fabiana C., Analysis of IEEE 802.15.4 Sensor Networks for Event Detection.

[3] Sofie, P., Mustafa, E., Sinem, C. E., Bruno B., Liesbet Van d., Francky, C., Ingrid, M., Ahmad B., Pravin V., Performance Analysis of Slotted IEEE 802.15.4 Medium Access Layer.

[4] Timmons, N.F. and Scanlon, W.G. 2004. Analysis of the performance of IEEE 802.15.4 for medical sensor body area networking, Proc. IEEE Communications Society Conference on Sensor and Ad Hoc Communications and Networks(SECON'04), pp. 16-24.

[5] Sun, Y. and Sun, Y. and Xu P. and Liu, H. 2013 Performance Analysis of Wireless Sensor Network Based on OPNET, Communications and Network, Vol. 5 No. 3B, pp. 512-516.

[6] L. Chaari, L. Kamoun, 2011. Performance analysis of IEEE 802.15.4/ Zigbee standard under real time constraints, International Journal of Computer Networks and Communication (IJCNC), Vol.3, No.5,pp. 235-251.

[7] Leung, S., Gomez, W. and Kim, J. J. 2009. ZigBee Mesh Network Simulation using OPNET and Study of Routing Selection.

[8] García-Hernández, C. F., Ibargüengoytia-González, P. H., García-Hernández, J. and Perez Diaz, J. A. 2007. Wireless Sensor Networks and Applications: a Survey", International Journal of Computer Science and Network Security, VOL.7 No.3.

[9] Boris Mihajlov1 and Mitko Bogdanoski2 , 2011. Overview and Analysis of the Performances of ZigBee based Wireless Sensor Networks, International Journal of Computer Applications, Volume 29- No.12.

[10] Shih, B., Chen, C., Chih, C. and Tseng, J. 2010. The development of enhancing mechanisms for improving the performance of IEEE 802.15.4", International Journal of the Physical Sciences Vol.5 (6), pp.884-897. 